University of Nebraska - Lincoln

DigitalCommons@University of Nebraska - Lincoln

Uniformed Services University of the Health

Sciences

U.S. Department of Defense

2011

\title{
Arachidonyl trifluoromethyl ketone ameliorates experimental autoimmune encephalomyelitis via blocking peroxynitrite formation in mouse spinal cord white matter
}

\author{
Adam C. Vana \\ Uniformed Services University of the Health Sciences \\ Shihe Li \\ Uniformed Services University of the Health Sciences \\ Rachel Ribeiro \\ Uniformed Services University of the Health Sciences \\ Flaubert Tchantchou \\ Uniformed Services University of the Health Sciences \\ Yumin Zhang \\ Uniformed Services University of the Health Sciences, yzhang@usuhs.mil
}

Follow this and additional works at: https://digitalcommons.unl.edu/usuhs

Part of the Medicine and Health Sciences Commons

Vana, Adam C.; Li, Shihe; Ribeiro, Rachel; Tchantchou, Flaubert; and Zhang, Yumin, "Arachidonyl trifluoromethyl ketone ameliorates experimental autoimmune encephalomyelitis via blocking peroxynitrite formation in mouse spinal cord white matter" (2011). Uniformed Services University of the Health Sciences. 43.

https://digitalcommons.unl.edu/usuhs/43

This Article is brought to you for free and open access by the U.S. Department of Defense at DigitalCommons@University of Nebraska - Lincoln. It has been accepted for inclusion in Uniformed Services University of the Health Sciences by an authorized administrator of DigitalCommons@University of Nebraska Lincoln. 
Regular Article

\title{
Arachidonyl trifluoromethyl ketone ameliorates experimental autoimmune encephalomyelitis via blocking peroxynitrite formation in mouse spinal cord white matter
}

\author{
Adam C. Vana ${ }^{\text {a }}$, Shihe Li $^{\text {a }}$, Rachel Ribeiro ${ }^{\text {b }}$, Flaubert Tchantchou ${ }^{\text {a }}$, Yumin Zhang ${ }^{\text {a,b,* }}$ \\ a Department of Anatomy, Physiology and Genetics, Uniformed Services University of the Health Sciences, 4301 Jones Bridge Road, Bethesda, MD 20814, USA \\ b Program in Neuroscience, Uniformed Services University of the Health Sciences, 4301 Jones Bridge Road, Bethesda, MD 20814, USA
}

\section{A R T I C L E I N F O}

\section{Article history:}

Received 10 November 2010

Revised 4 May 2011

Accepted 22 May 2011

Available online 13 June 2011

\section{Keywords:}

Phospholipase $\mathrm{A}_{2}$

Peroxynitrite

Inducible nitric oxide synthase

NADPH oxidase

Microglia

Oligodendrocytes

Experimental autoimmune encephalomyelitis

\begin{abstract}
A B S T R A C T
Inhibition of phospholipase $\mathrm{A}_{2}\left(\mathrm{PLA}_{2}\right)$ has recently been found to attenuate the pathogenesis of experimental autoimmune encephalomyelitis (EAE), a commonly used animal model of multiple sclerosis (MS). However, the protective mechanisms that underlie $\mathrm{PLA}_{2}$ inhibition are still not well understood. In this study, we found that cytosolic $\mathrm{PLA}_{2}\left(\mathrm{CPLA}_{2}\right)$ was highly expressed in infiltrating lymphocytes and macrophages/microglia in mouse spinal cord white matter. Although $\mathrm{CPLA}_{2}$ is also expressed in spinal cord neurons and oligodendrocytes, there were no differences observed in these cell types between EAE and control animals. Arachidonyl trifluoromethyl ketone (AACOCF3), a cPLA 2 inhibitor, significantly reduced the clinical symptoms and inhibited the body weight loss typically found in EAE mice. AACOCF3 also attenuated the loss of mature, myelin producing, oligodendrocytes, and axonal damage in the spinal cord white matter. Nitrotyrosine immunoreactivity, an indicator of peroxynitrite formation, was dramatically increased in EAE mice and attenuated by treatment with AACOCF3. These protective effects were not evident when AA861, an inhibitor of lipoxygenase, was used. In primary cultures of microglia, lipopolysaccharide (LPS) induced an upregulation of $\mathrm{CPLA}_{2}$, inducible nitric oxide synthase (iNOS) and components of the NADPH oxidase complex, p47phox and p67phox. AACOCF3 significantly attenuated iNOS induction, nitric oxide production and the generation of reactive oxygen species in reactive microglia. Similar to the decomposition catalyst of peroxynitrite, AACOCF3 also blocked oligodendrocyte toxicity induced by reactive microglia. These results suggest that AACOCF3 may prevent oligodendrocyte loss in EAE by attenuating peroxynitrite formation in the spinal cord white matter.

Published by Elsevier Inc.
\end{abstract}

\section{Introduction}

Experimental autoimmune encephalomyelitis (EAE) is a well recognized animal model for multiple sclerosis (MS), which is characterized by lymphocyte infiltration, macrophages/microglia activation, demyelination, oligodendrocyte (OL) loss and axonal injury (Bjartmar et al., 2003; Furlan et al., 2009; Lassmann, 2010; Steinman and Zamvil, 2006). Although a number of pharmacological agents have shown promise in the treatment of EAE, the pathogenic mechanisms of MS/EAE and the therapeutic targets of the drugs' action are still not well understood.

Activation of phospholipase $\mathrm{A}_{2}\left(\mathrm{PLA}_{2}\right)$ has recently emerged as an important initiator in the pathogenesis of many inflammatory and neurodegenerative diseases, including MS and Alzheimer's disease (Farooqui et al., 2006; Sanchez-Mejia et al., 2008; Shelat et al., 2008). $\mathrm{PLA}_{2}$ occurs as three isozymes. These isoforms include calcium-

\footnotetext{
* Corresponding author at: Department of Anatomy, Physiology and Genetics, Uniformed Services University of the Health Sciences, 4301 Jones Bridge Road, Bethesda, MD 20814, USA. Fax: + 13012953566.

E-mail address: yzhang@usuhs.mil (Y. Zhang).
}

dependent cytosolic PLA P $_{2}\left(\mathrm{CPA}_{2}\right)$, secretory PLA $\mathrm{P}_{2}\left(\mathrm{sPLA}_{2}\right)$ and calcium-independent $\mathrm{PLA}_{2}$ (iPLA ${ }_{2}$ ) (Farooqui and Horrocks, 2006; Kudo and Murakami, 2002). Of the three PLA 2 isoforms, CPLA preferentially hydrolyzes phospholipids containing arachidonic acids (Kita et al., 2006; Kudo and Murakami, 2002) and plays a key role in the biosynthesis of prostaglandins and leukotrienes via activation of cyclooxygenase (COX) and lipoxygenase (LOX), respectively (Funk, 2001). Although several COX inhibitors have been shown to ameliorate EAE (Muthian et al., 2006; Ni et al., 2007), the severe side effects caused by these inhibitors, such as gastrointestinal ulcers triggered by indomethacin (Miyamoto et al., 2006) and the cardiovascular risk induced by rofecoxib (Juni et al., 2004), suggest that COX inhibitors might not be practically useful for the treatment of MS (James et al., 2007). Considering the fact that inhibition of COX can shift the arachidonic acid metabolism from the COX pathway to the LOX pathway, it is possible that inhibitors of LOX or the combined inhibition of PLA 2 and LOX could directly lead to better therapeutic outcomes.

We have previously shown that peroxynitrite, the toxic species released from reactive microglia (Li et al., 2005), causes OL toxicity by activation of 12-LOX (Zhang et al., 2006). Since the production of 
arachidonic acid from membrane phospholipids catalyzed by cPLA $\mathrm{A}_{2}$ is the rate-limiting step for the subsequent 12-LOX metabolism, blocking $\mathrm{CPLA}_{2}$ and LOX activation may prevent OL toxicity in an animal model of MS. Using myelin oligodendrocyte glycoprotein (MOG) 35-55 peptide-induced EAE mouse model, we found that AACOCF3, a CPLA 2 inhibitor, but not AA861, a LOX inhibitor, ameliorates the clinical signs of EAE. The protective mechanisms of AACOCF3 might be due to its improvement of blood-brain barrier function and its inhibition of peroxynitrite formation in EAE mouse spinal cord white matter.

\section{Materials and methods}

\section{Materials}

AACOCF3 was obtained from Cayman Chemical Co. (Ann Arbor, MI). Fe (III) tetrakis(1-methyl-4-pyridyl) porphyrin pentachlorideporphyrin pentachloride (FeTMPyP) was obtained from Calbiochem (San Diego, CA). 2',7'-dichlorohydrofluorescein diacetate (DCF) was purchased from Molecular Probes, Inc. (Eugene, OR). All the culture materials were purchased from Gibco Life Technologies (Grand Island, NY). Platelet-derived growth factor (PDGF), basic fibroblast growth factor (bFGF), and ciliary neurotrophic factor (CNTF) were purchased from Peprotech (Princeton, NJ). All other reagents were obtained from Sigma (St. Louis, MO).

Mice

Female 7-week-old C57Bl/6J mice were purchased from The Jackson Laboratory (Bar Harbor, ME). Animal care and experimental procedures were carried out in accordance with $\mathrm{NIH}$ guidelines and approved by the Uniformed Services University Animal Care and Use Committee.

\section{Induction and clinical evaluation of EAE}

EAE was induced by subcutaneous injection of $200 \mu \mathrm{g}$ of MOG 35-55 peptide emulsified in Complete Freunds Adjuvant (CFA, DIFCO) with $500 \mu \mathrm{g}$ Mycobacterium tuberculosis (DIFCO). Immediately following MOG peptide injection, and $24 \mathrm{~h}$ later, mice were administered $200 \mathrm{ng}$ pertusis toxin (List Biological Labs) intraperitoneally (i.p.). At 1 week after induction, EAE mice received a booster of $200 \mu \mathrm{g}$ of MOG in incomplete Freund's Adjuvant without Mycobacterium tuberculosis. Animals were monitored blindly and daily by two independent observers and neurological signs were assessed as follows: 0 , normal mouse; 1 , piloerection, tail weakness; 2 , tail paralysis; 3 , tail paralysis plus hindlimb weakness/paralysis; 4 , tail, hind and fore limb paralysis; 5 , moribund/death, using increments of 0.5 points for intermediate clinical findings. The onset of EAE was defined as the first day an animal showed a clinical score $\geq 0.5$.

\section{Drug treatment}

EAE and control mice were randomly assigned to receive AACOCF3 (4 mM, Cayman), a cPLA 2 inhibitor; AA861 (4 mM, Sigma), a LOX inhibitor; AACOCF3 plus AA861; or vehicle (1\% DMSO in PBS). There were 6 animals in each group. Drug treatments were given at day 7 post-immunization (i.p.) daily until day 28. A second set of experiments were similarly designed, except for the animals were sacrificed at day 14 post-immunization for assessing the inflammatory response in the spinal cord white matter of EAE with/without treatments.

\section{Immunohistochemistry (IHC)}

Animals were euthanized using a combination of ketamine and xylazine solution (90 mg ketamine/10 mg xylazine per ml, i.p.), then intracardially perfused with ice cold $1 \mathrm{M}$ phosphate buffer followed by $4 \%$ paraformaldehyde (Sigma, St. Louis, MO) in $1 \mathrm{M}$ phosphate buffer. Spinal cords were dissected out prior to post-fixation in $4 \%$ paraformaldehyde at $4{ }^{\circ} \mathrm{C}$ overnight. Tissue was then cryoprotected in $30 \%$ sucrose (Sigma) in $1 \mathrm{M}$ phosphate buffer at $4{ }^{\circ} \mathrm{C}$ overnight. After being cryoprotected the tissue was embedded in Tissue Tek OCT (Sakura, Torrance, CA) and stored at $-80{ }^{\circ} \mathrm{C}$ until utilization. Transverse sections of lumbar spinal cords were cut at $14 \mu \mathrm{m}$ with a cryostat (Leica model CM1900, Bannockburn, IL) and mounted onto Superfrost Plus slides (Fisher, Pittsburgh, PA) for immunohistological analysis. To determine the cell type specific expression of $\mathrm{CPLA}_{2}, 5$ LOX and 12-LOX, we performed double immunostaining using polyclonal antibodies against CPLA $_{2}$ (Santa Cruz Biotechnology, Santa Cruz, CA), 5-LOX and 12-LOX (Cayman Chemicals, Ann Arbor, $\mathrm{MI}$ ), and monoclonal antibodies against CC1 (Calbiochem, San Diego, CA), NeuN (Millipore, Billerica, MA), OX52 (Abcam, Cambridge, MA) and Mac1 (Millipore, Billerica, MA), markers for mature OLs, neurons, T-lymphocytes and macrophages/microglia, respectively. Nitrotyrosine reactivity in spinal cord white matter was examined using a polyclonal anti-nitrotyrosine antibody (Millipore, Billerica, MA). Neurofilaments were labeled with NF-200 (Millipore, Billerica, MA). SMI-32 (Abcam, Cambridge, MA) was used to label the dephosphorylated neurofilaments, indicative of demyelination and axonal injury (Trapp et al., 1998). Beta-amyloid precursor protein ( $\beta$-APP) was also used to detect the degenerated axons. Activated leukocyte cell adhesion molecule (ALCAM) and intercellular adhesion molecule1 (ICAM-1) were detected with anti-ALCAM and anti-ICAM polyclonal antibodies (Santa Cruz Biotechnology, Santa Cruz, CA).

\section{Cell cultures}

Primary cultures of microglia and OLs were prepared from the cerebral hemispheres of Sprague-Dawley rats at postnatal day 2 using a selective detachment procedure as previously described (Wang et al., 2004; Zhang et al., 2006). Briefly, forebrains free of meninges were dissected out and digested with Hank's balanced salt solution (HBSS) containing $0.01 \%$ trypsin and $10 \mathrm{mg} / \mathrm{ml}$ DNase. After centrifugation and trituration with the plating medium DMEM20S containing DMEM, 20\% fetal bovine serum, and 1\% penicillin-streptomycin, cells were plated onto polylysine coated $75-\mathrm{cm}^{2}$ flasks at a density of 1 pup brain per flask. Cultures were fed with fresh DMEM20S medium every other day for $10-11$ days at $37^{\circ} \mathrm{C}$ in a humid atmosphere of $5 \%$ $\mathrm{CO}_{2} / 95 \%$ air. Microglia were isolated by gently shaking the flask containing the mixed glial culture for $1 \mathrm{~h}$ at $200 \mathrm{rpm}$ at $37^{\circ} \mathrm{C}$. The medium was removed, and microglia were collected by centrifugation at $800 \times \mathrm{g}$ for $5 \mathrm{~min}$. Isolated microglia were plated into 24 -well $\left(5 \times 10^{4}\right.$ per well $)$ or 96 -well $\left(1 \times 10^{4}\right.$ per well $)$ plates and experiments were performed in the next 2 days. The purity of the microglia cultures was consistently $>95 \%$.

After removing microglia, the flasks were then changed to new media and shaken overnight. The OL progenitor cells were detached from the astrocyte layer and were resuspended and seeded onto polyornithine coated 24 -well plates $\left(4 \times 10^{5}\right.$ cells/plate) in a basal chemically-defined medium (BDM) [DMEM with $1 \mathrm{mg} / \mathrm{ml}$ bovine serum albumin (BSA), $50 \mu \mathrm{g} / \mathrm{ml}$ apo-transferrin, $5 \mu \mathrm{g} / \mathrm{ml}$ insulin, $30 \mathrm{nM}$ sodium selenite, $10 \mathrm{nM}$ biotin, $10 \mathrm{nM}$ hydrocortisone] plus $10 \mathrm{ng} / \mathrm{ml}$ of both PDGF and bFGF. The cells were maintained in BDM and the medium was half-changed 3 times per week. For culturing mature OLs, at day 7 OLs were changed to BDM plus 3,3',5-triiodo-Lthyronine $\left(\mathrm{T}_{3}\right)(15 \mathrm{nM})$ and $\mathrm{CNTF}(10 \mathrm{ng} / \mathrm{ml})$ and were half-changed 3 times per week for 2 weeks. At this stage, more than 95\% of the cells were MBP positive (Baud et al., 2004; Wang et al., 2004) and were 


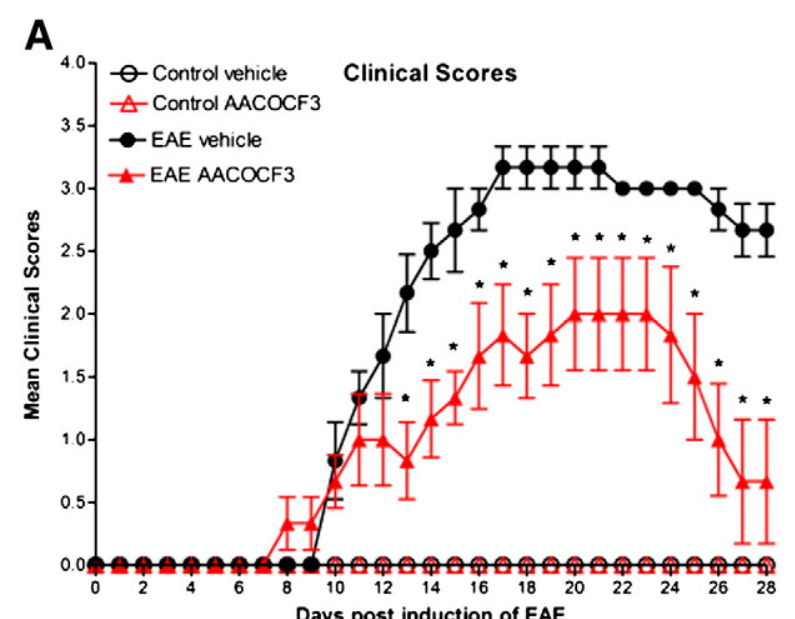

Days post induction of EAE

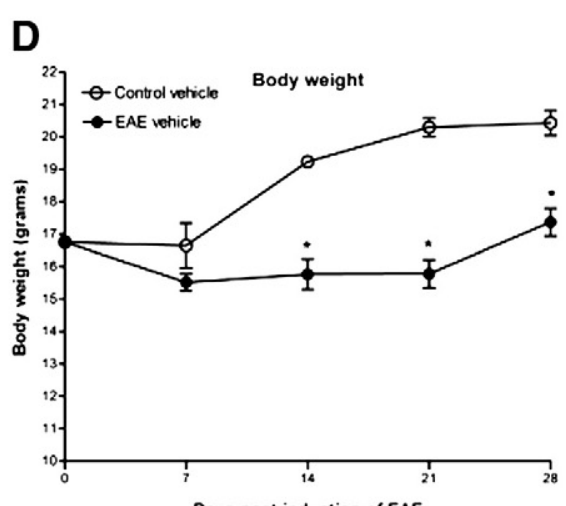

E

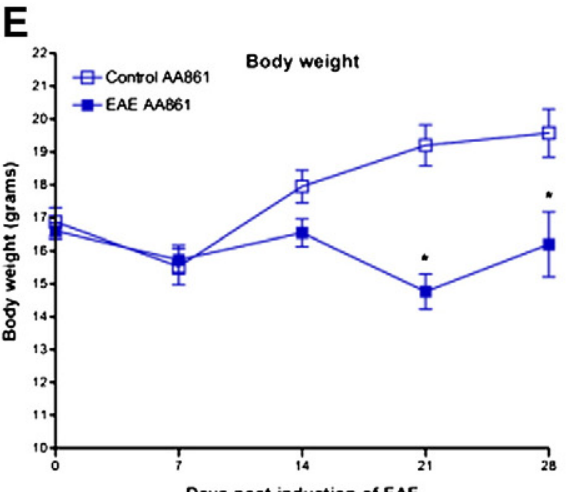

Days post induction of EAE

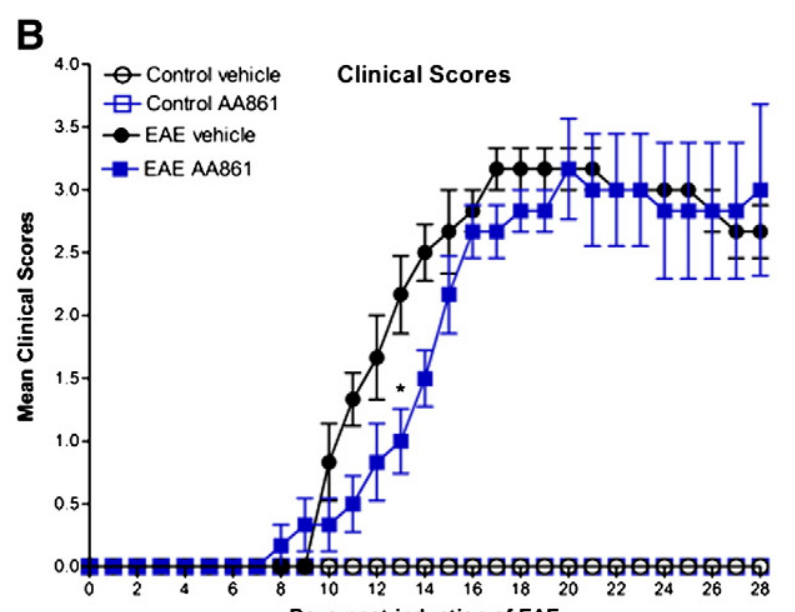

Days post induction of EAE
C
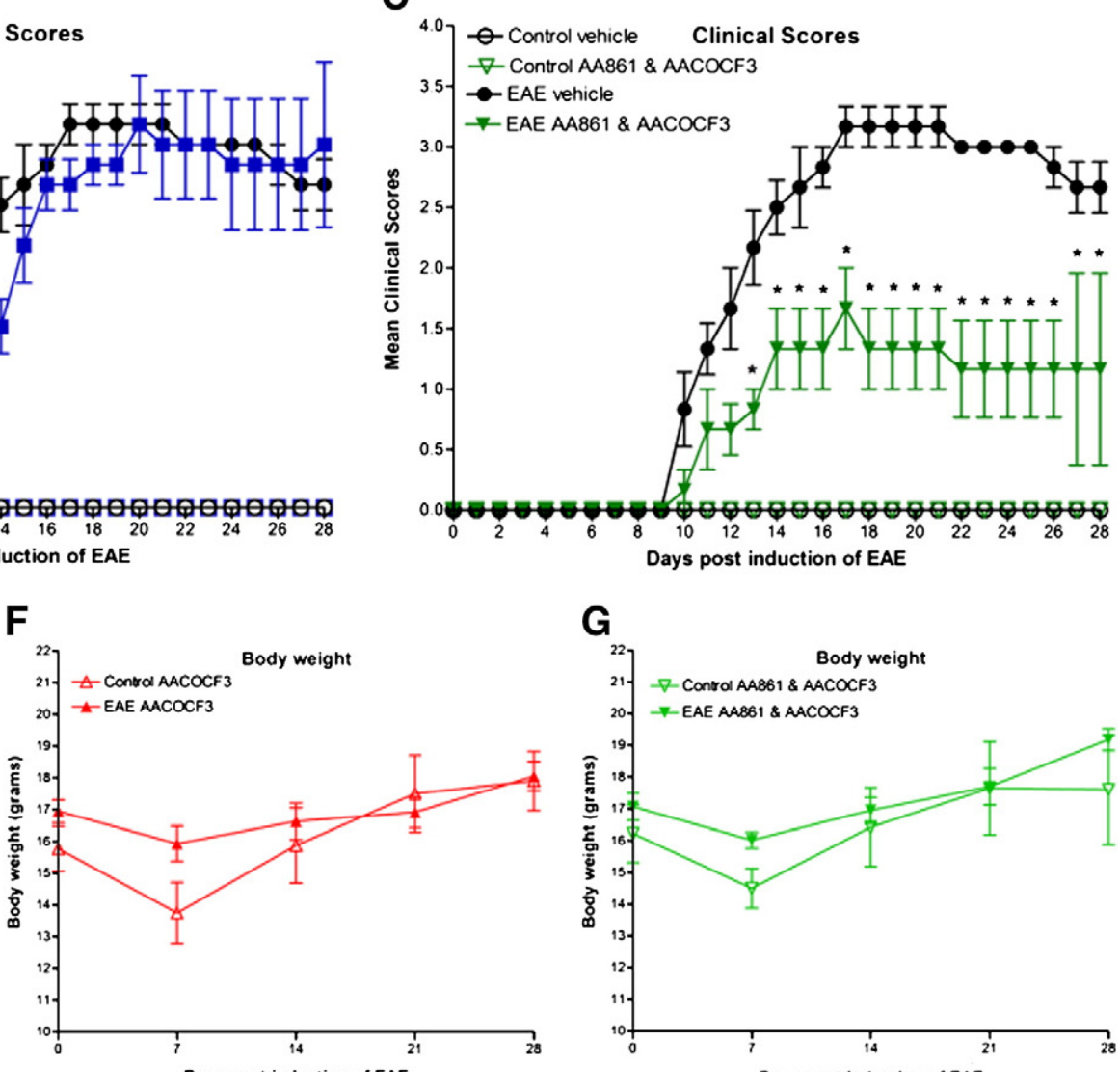

Days post induction of EAE

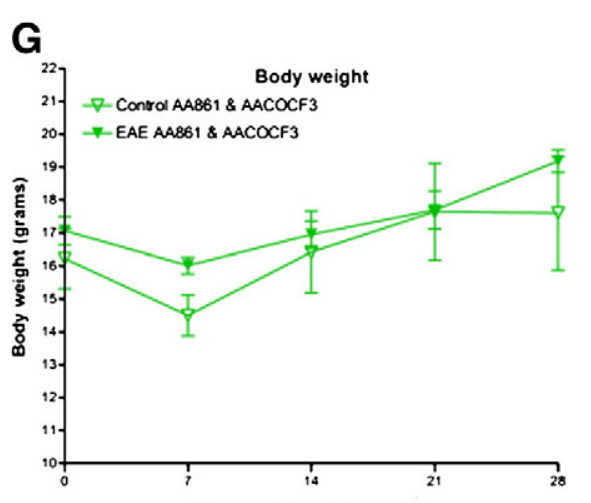

Days post induction of EA

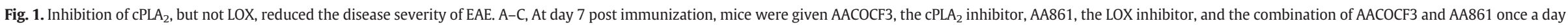

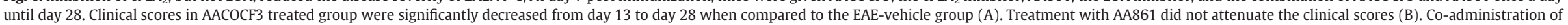

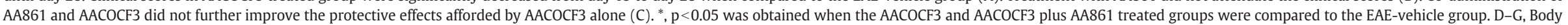

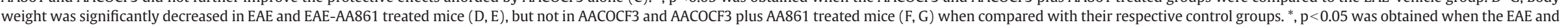
EAE-AA861 treated groups were compared to the mice given vehicle and AA861 control groups. 
used for the experiments. The contamination of astrocytes and microglia was $1-2 \%$ each. For mixed cultures of microglia and OLs, microglia were plated to 24-well plates containing mature OLs and then used for further experiments.

\section{Western blot analyses}

At various time points after LPS treatment, microglia were lysed with lysis buffer containing $20 \mathrm{mM}$ Tris- $\mathrm{HCl}, \mathrm{pH} 7.5,150 \mathrm{mM} \mathrm{NaCl}$,
$1 \mathrm{mM}$ EDTA, 1 mM EGTA, 1\% Triton X-100, $2.5 \mathrm{mM}$ sodium pyrophosphate, $1 \mathrm{mM}$ glycerolphosphate, $1 \mathrm{mM} \mathrm{Na}_{3} \mathrm{VO}_{4}$, and $1 \mathrm{mM}$ phenylmethylsulfonyl fluoride. An aliquot of cell lysate was removed for later protein determination. Cell lysate was mixed with Laemmli buffer, boiled for $5 \mathrm{~min}$, and stored at $-20^{\circ} \mathrm{C}$. Equal amounts of protein were separated by $4-12 \%$ SDS-PAGE and electrotransferred to a polyvinylidene difluoride membrane. Membranes were blocked with $5 \%$ nonfat milk in Tris-buffered saline containing $0.1 \%$ Tween 20 (TBST) for $1 \mathrm{~h}$ and then incubated overnight at $4{ }^{\circ} \mathrm{C}$ with the primary antibodies
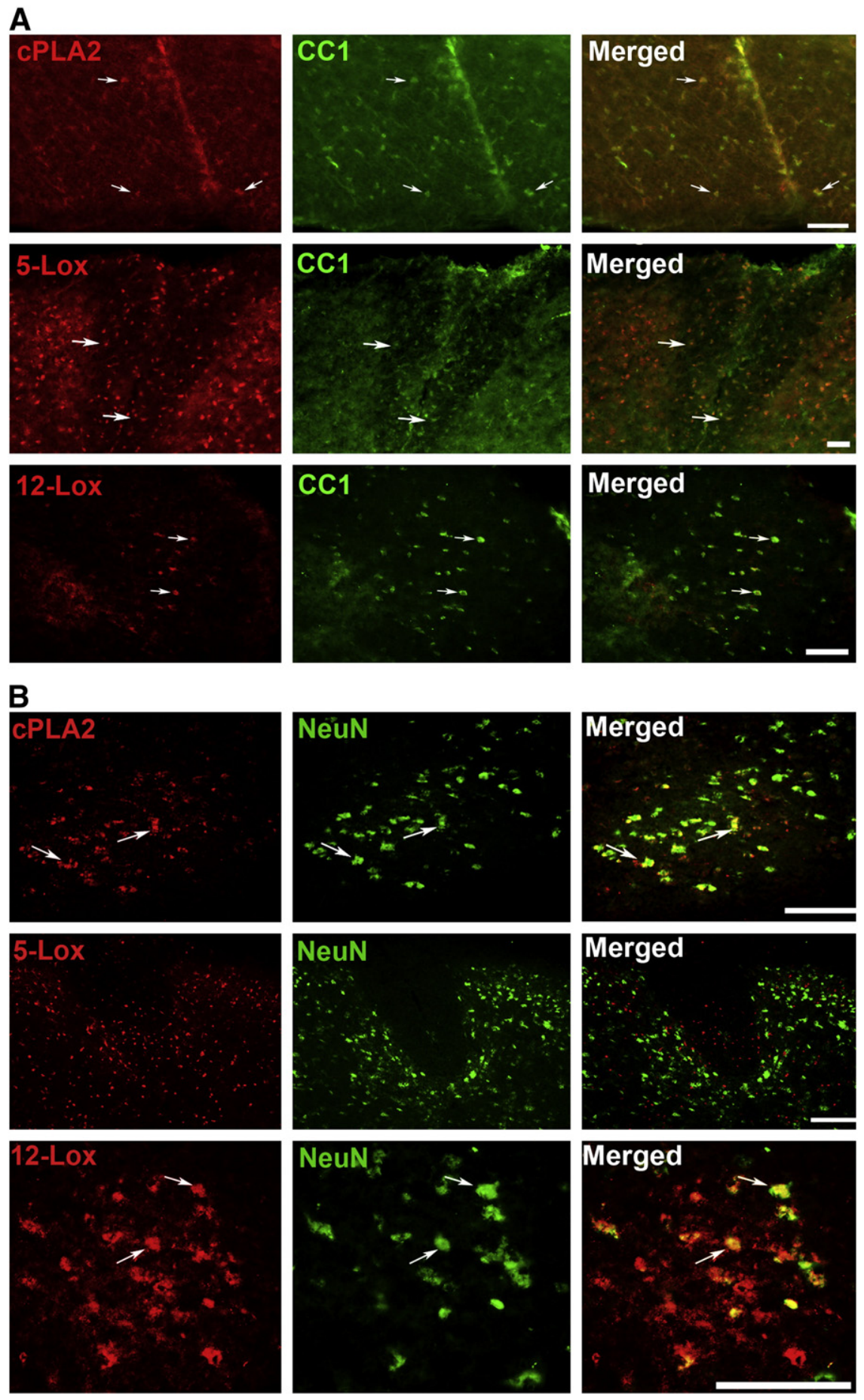

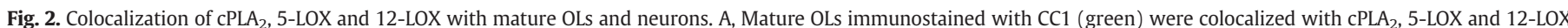

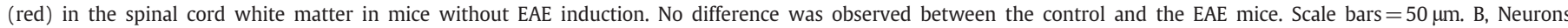

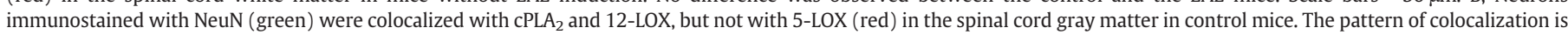
the same in both control and EAE mice. Scale bars $=100 \mu \mathrm{m}$. 
against cPLA ${ }_{2}$ (Santa Cruz Biotechnology, Santa Cruz, CA), iNOS, p47phox, p67phox (BD Biosciences Pharmingen) and nitrotyrosine (Upstate Biotechnology, Lake Placid, NY) diluted at 1:1000 in TBST containing 5\% BSA. After washing 4 times with TBST, the membrane was incubated for $1 \mathrm{~h}$ at room temperature with goat anti-rabbit and goat anti-mouse horseradish peroxidase-conjugated secondary antibodies (Santa Cruz Biotechnology, Santa Cruz, CA) diluted at 1:2000. The membranes were washed again as above and visualized by enhanced chemiluminescence (ECL) according to the manufacturer's protocol (PerkinElmer Life Sciences).

\section{Immunocytochemistry and immunofluorescence microscopy}

After treatments, cells were fixed with $4 \%$ paraformaldehyde in PBS for $10 \mathrm{~min}$ at room temperature, washed three times with PBS, and blocked with TBST (50 mM Tris-HCl, pH 7.4/150 mM NaCl/0.1\% Triton X-100) containing $5 \%$ goat serum for $1 \mathrm{~h}$ at room temperature. The coverslips were incubated with mouse monoclonal antibodies against $\mathrm{CPLA}_{2}$, p67phox, MBP and polyclonal antibody against F4/80 overnight at $4{ }^{\circ} \mathrm{C}$. After three to four washes at $5 \mathrm{~min}$ each, the appropriate secondary antibody conjugated with either Alexia Fluor 488 or Alexia Fluor 594 (Molecular Probes) was added to the coverslips and incubated for $1 \mathrm{~h}$. After three washes, the coverslips were mounted onto glass slides with FluoroMount and kept dark at $4{ }^{\circ} \mathrm{C}$. Cell images were captured with a fluorescence microscope (Nikon Eclipse E800) equipped with a Nikon digital camera.

\section{Nitrite measurement}

The level of accumulated nitrite in the medium was determined by the Greiss reaction. Briefly, $50 \mu \mathrm{l}$ of Greiss reagent $(3.9 \mathrm{mM} \mathrm{N}-(1-$ naphthyl)ethylenediamine $/ 58 \mathrm{mM}$ sulfanilamide $/ 5 \%$ phosphoric acid) was added to $50 \mu \mathrm{l}$ of culture supernatant in a 96-well plate. Absorbance was measured at $550 \mathrm{~nm}$, and nitrite concentration was calculated from a standard curve of sodium nitrite.

\section{Measurement of intracellular ROS generation}

Intracellular free radical generation was evaluated with DCF (Molecular Probes, Eugene, OR) as we previously described (Wang et al., 2004; Zhang et al., 2006). Briefly, after cells in 96-well plates were treated with LPS in the absence or presence of AACOCF3 for $3 \mathrm{~h}$, they were then loaded with DCF $(20 \mu \mathrm{M})$ for $30 \mathrm{~min}$ in Earle's balanced salt solution (EBSS; $95 \%$ air $/ 5 \% \mathrm{CO}_{2}, 37^{\circ} \mathrm{C}$ ). After the loading solution was removed, cells in the wells were washed and incubated in EBSS. The fluorescence of the cells in each well was measured and recorded in the fluorescence plate-reader with excitation wavelength at $480 \mathrm{~nm}$ and emission wavelength at $530 \mathrm{~nm}$.

\section{Toxicity assay}

Survival of mature OLs was determined by counting $\mathrm{MBP}^{+}$cells with normal nuclei. Briefly, the mixed cultures of microglia and OLs were treated with LPS ( $5 \mu \mathrm{g} / \mathrm{ml})$ as specified in the figure legends for $48 \mathrm{~h}$. After being washed with PBS and fixed with $4 \%$ paraformaldehyde, cells were immunostained with anti-MBP (Boehringer Mannheim, Indianapolis, IN) and anti-F4/80 (eBiosciences) antibodies. Five random fields were counted in each coverslip under $20 \times$ magnification.

\section{Statistics}

Cell densities in lumbar spinal cord white matter were manually counted with areas measured using Nikon Elements software. Statistical significance in vitro and in vivo assays was assessed using ANOVA with the Tukey-Kramer post-hoc multiple comparison test. Statistical analysis was performed using the Instat program from GraphPad Software (San Diego, CA). Representative experiments are shown unless noted otherwise. Experiments were performed with triplicate samples, and the data are expressed as mean \pm SEM. All experiments were repeated at least 3 times.

\section{Results}

Treatment with a PLA $A_{2}$ inhibitor, but not a LOX inhibitor, reduced the severity of $E A E$

It is currently unclear how activation of LOX is involved in the development of EAE. Although a LOX inhibitor has recently been shown to delay the disease onset and the cumulative clinical scores of
A
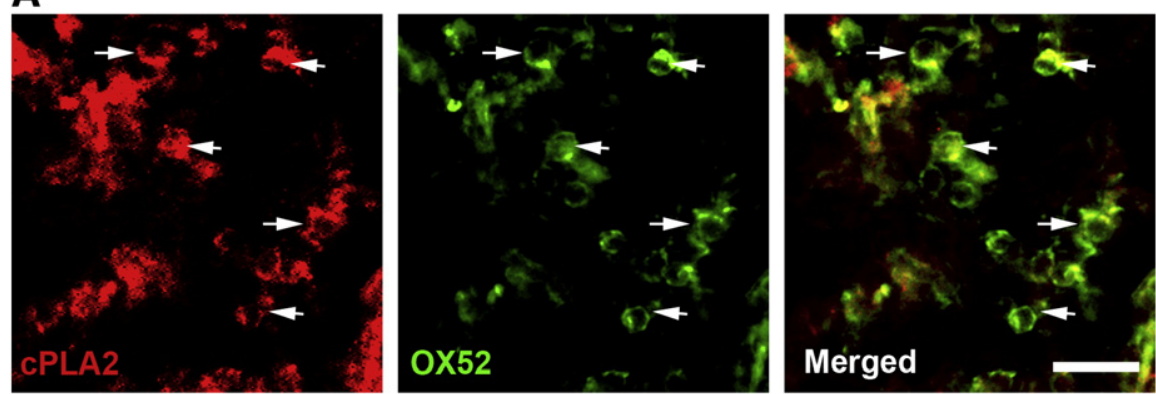

B

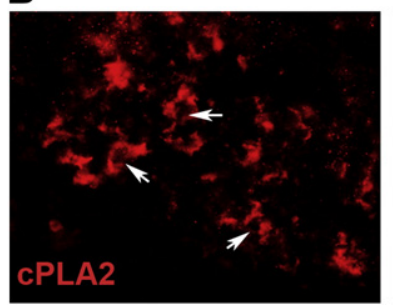

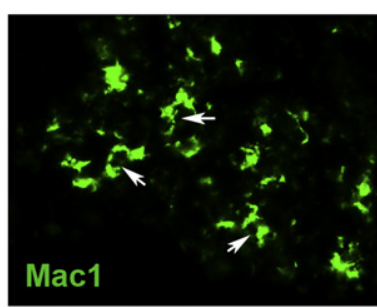

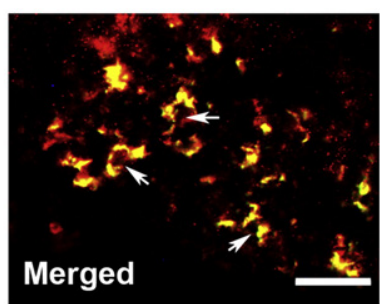

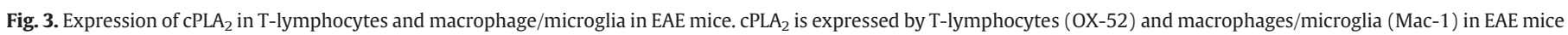
at 2 weeks post immunization. Images shown are representative from four different animals in each group. Scale bars $=25 \mu \mathrm{m}$. 
EAE, the maximal clinical score was not affected by the treatment (Marusic et al., 2008). Moreover, similar to the results obtained from COX-2 knockout (COX-2 $2^{-/-}$) mice (Miyamoto et al., 2006), targeted gene deletion of 5-LOX and 12-LOX does not affect, but actually exacerbates the severity of EAE (Emerson and LeVine, 2004). To further determine the role of 5-LOX or 12-LOX in the pathogenesis of EAE, we treated EAE mice with AA861, the LOX inhibitor, AACOCF3, the $\mathrm{CPLA}_{2}$ inhibitor, and the combination of both from day 7 postimmunization. Consistent with the reports by others (Kalyvas et al., 2009; Kalyvas and David, 2004; Marusic et al., 2005, 2008), AACOCF3 was found to significantly attenuate the clinical signs of EAE (Fig. 1A). Although there was a trend of delayed onset of the symptoms, significant differences were not reached when the clinical scores were compared between the AA861 and the vehicle treated EAE mice (Fig. 1B). Similarly, the combination of AA861 and AACOCF3 did not exert more protective effects (Fig. 1C) than AACOCF3 itself (Fig. 1A). A significantly decreased body weight was observed in the vehicle and AA861 treated mice, but not in the AACOCF3 treated EAE groups when compared to their respective controls (Fig. 1D-G). These results suggest that blocking the production of arachidonic acid, rather than its downstream LOX metabolites, is effective against the disease severity of EAE.

cPLA $A_{2}$ is highly expressed in T-lymphocytes and macrophages/microglia in the spinal cord white matter of mice with $E A E$

Using cell type specific markers, we examined the expression of $\mathrm{CPLA}_{2}, 5$-LOX and 12-LOX in the spinal cord mature OLs and neurons in both EAE and control mice. Similar to the findings from a previous report (Liu et al., 2006), we found that $\mathrm{CPLA}_{2}$ is expressed in both $\mathrm{CC}^{+}$mature OLs (Fig. 2A) and $\mathrm{NeuN}^{+}$neurons (Fig. 2B). While 12LOX was also shown to be expressed in both OLs and neurons, the expression of 5-LOX was seen in $\mathrm{CC}^{+}{ }^{+}$OLs (Fig. 2A), but not in $\mathrm{NeuN}^{+}$ cells (Fig. 2B). When the expression was examined at 2 weeks following EAE induction, a similar expression pattern was also observed in EAE mice (data not shown). These results suggest that CPLA $_{2}, 5$-LOX and 12-LOX were not upregulated in these resident cells of the spinal cord. Next, we assessed the expression of $\mathrm{CPLA}_{2}$ in infiltrating T-lymphocytes and macrophages/microglia using OX-52 and Mac1 as the respective markers of these cell types. As shown in
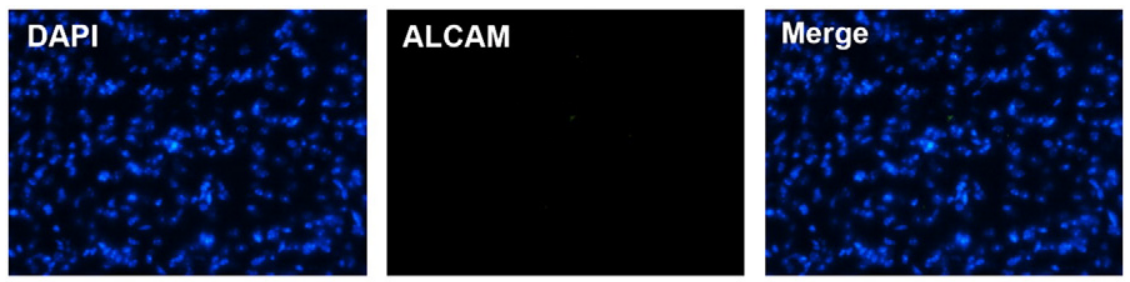

Naive
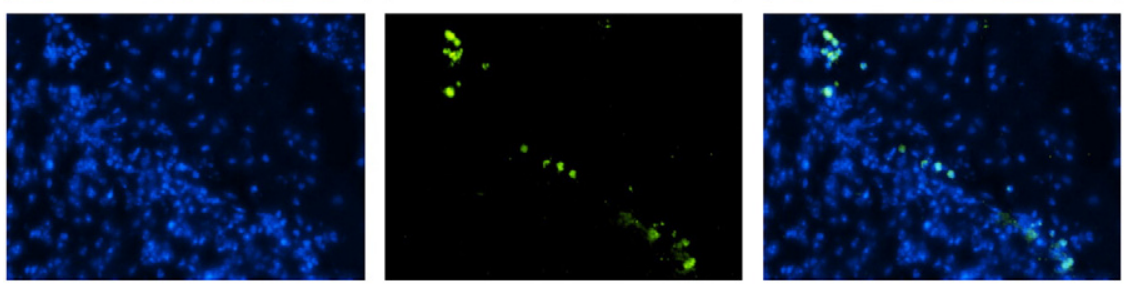

EAE 2 wk

Vehicle
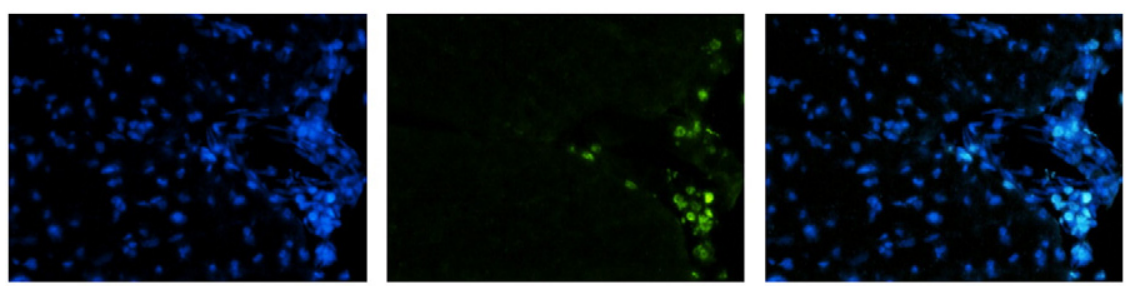

EAE 4 wk

Vehicle
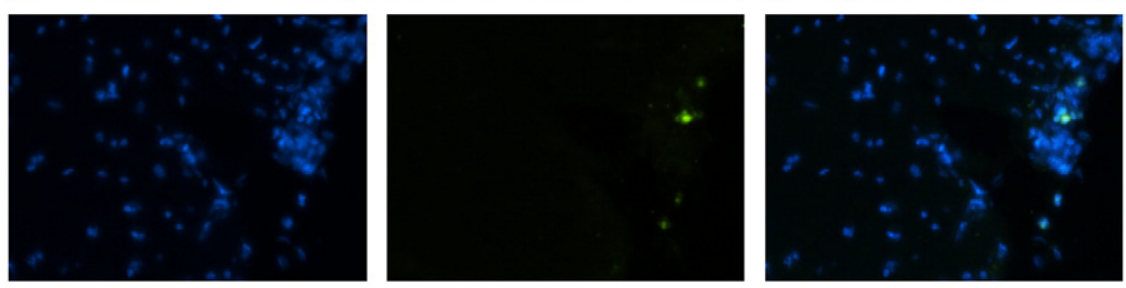

EAE 2 wk
AACOCF3
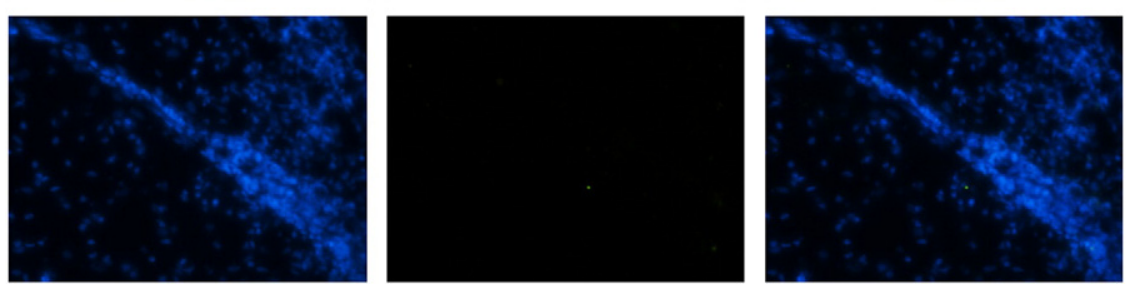

EAE 4 wk AACOCF3

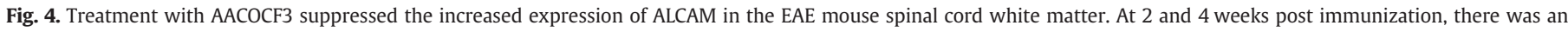

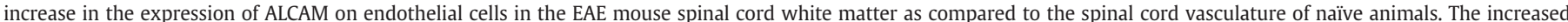

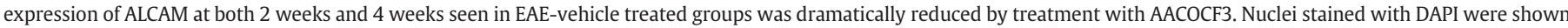

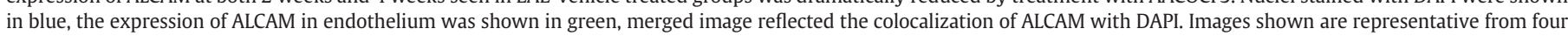
different animals in each group. 


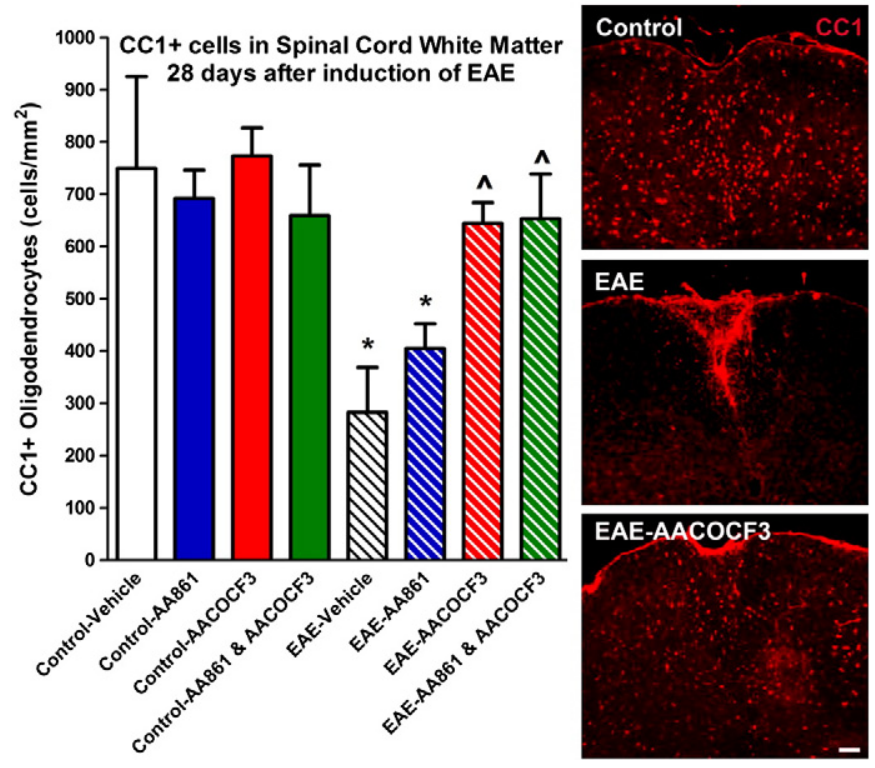

Fig. 5. Treatment with AACOCF3, but not AA861, completely blocked the loss of mature OLs in the EAE mouse spinal cord white matter. At 28 days post immunization, the density of mature OLs in the spinal cord white matter of EAE mice was significantly less than that found in the control animals. The loss of mature OLs was prevented by the administration of AACOCF3 alone or its combination with AA861, but not by the treatment with AA861. *, p $<0.05$ was obtained when the EAE-vehicle and EAE-AA861 treated groups were compared to the respective control groups. Increased density of OLs were found in AACOCF3 treated groups compared with EAE-vehicle group $(\wedge, \mathrm{p}<0.05)$. The representative image showing the $\mathrm{CC} 1^{+}$mature OLs in control, EAE and AACOCF3 treated mouse spinal cord white matter was shown. Scale bar $=50 \mu \mathrm{m}$.

Fig. 3, cPLA 2 is highly expressed in T-lymphocytes and macrophages/ microglia in EAE mice, but not in controls. These results suggest that the increased expression of $\mathrm{CPLA}_{2}$ in lymphocytes and macrophages/ microglia might play an important role in the immune response of EAE.

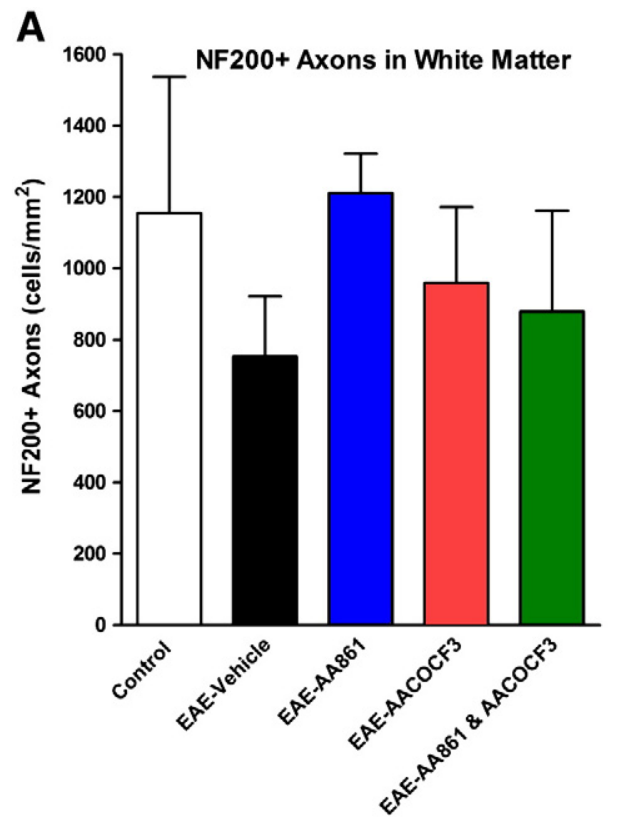

AACOCF3 preserved the integrity of blood-brain barrier after EAE induction

During an MS/EAE exacerbation, T-cells infiltration and macrophages/microglia activation are facilitated by an increased permeability of blood-brain barrier (BBB). To determine the integrity of BBB, we assessed the expression of ALCAM in EAE mice spinal cord white matter with/without treatment. ALCAM is now recognized as an adhesion molecule involved in lymphocyte and macrophage infiltration across the BBB. The expression of ALCAM on BBB endothelial cells has been shown to be upregulated by proinflammatory cytokines and enhanced in EAE spinal cord homogenates and in human MS lesions (Cayrol et al., 2008). Consistently, we found that the expression of ALCAM in the ventral spinal cord white matter was increased in EAE mice at 2 weeks and 4 weeks post-induction, and the increased expression is reduced or diminished by treatment with AACOCF3 (Fig. 4). The same phenomenon was also found with ICAM-1, a well characterized adhesion molecule expressed on inflamed BBB endothelial cells (Alvarez et al., 2011; Chaudhary et al., 2006) (data not shown). These results suggest that treatment with AACOCF3 may preserve the BBB integrity in EAE mice.

Treatment with AACOCF3 alone or in combination with AA861 completely prevented the loss of mature OLs and the axonal injury

A significant loss of mature OLs was found at 28 days following EAE induction in the spinal cord white matter of the vehicle treated mice (Fig. 5). The number of mature OLs in the EAE vehicle treated animals was $290 \pm 85$ cells $/ \mathrm{mm}^{2}$, which was significantly less than the number of mature OLs in controls $\left(750 \pm 180\right.$ cells $\left./ \mathrm{mm}^{2}, \mathrm{p}<0.05\right)$. In AACOCF3 alone and its combination with AA861 treated groups, the numbers of mature OLs increased to $650 \pm 37$ cells $/ \mathrm{mm}^{2}$ and $655 \pm 86$ cells $/ \mathrm{mm}^{2}$, respectively. The density of mature OLs in these groups is significantly higher $(\mathrm{p}<0.05)$ than the non-treated EAE group, and is similar to that of the control groups. On the other hand, treatment with AA861 did not prevent the loss of mature OLs. The density of OLs

B

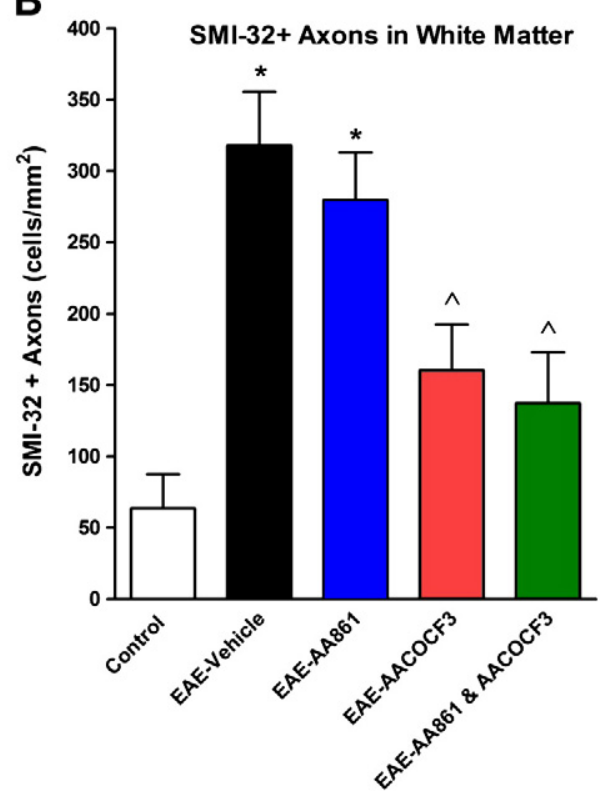

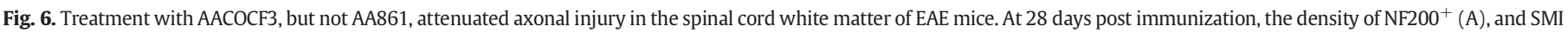

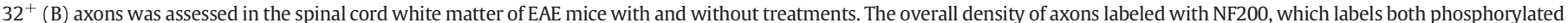

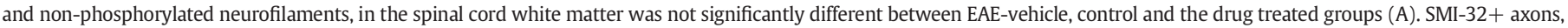

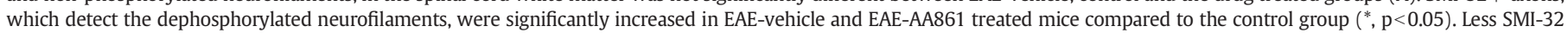
staining was shown in AACOCF3 treated groups compared to EAE-vehicle group ( $\left.{ }^{\wedge}, \mathrm{p}<0.05\right)(B)$. The results shown are pooled from 6 different animals in each group. 
in the AA861 treated group was $400 \pm 38$ cells $/ \mathrm{mm}^{2}$, which was similar in the untreated EAE animals.

To determine whether the drug treatment could affect axonal injury, the number of $\mathrm{NF} 200^{+}$axons in spinal cord white matter was counted. Although there was a trend of reduction of NF200 ${ }^{+}$axons in EAE-vehicle group, the overall density of $\mathrm{NF} \mathrm{OO}^{+}$axons was not significantly different in the control and the EAE groups regardless of the drug treatments (Fig. 6A). Because NF-200 can detect both phosphorylated and non-phosphorylated axonal neurofilaments, the differences in the number of non-/de-phosphorylated axons among these groups might be masked, unless the dephosphorylated axons can be rapidly degraded. However, SMI-32, which detects dephosphorylated neurofilaments, is an indicator of axonal injury in MS and EAE (Trapp et al., 1998). We found that the density of SMI- $32^{+}$axons was significantly increased in vehicle and AA861 treated EAE groups. Treatment with AACOCF3, or the combination of AACOCF3 and AA861, remarkably reduced the number of SMI- $32^{+}$axons (Fig. 6B). Similarly, increased $\beta$-APP immunoreactivity in spinal cord white matter was also reduced by treatment with AACOCF3 (data not shown). Taken together, these results suggest that treatment with AACOCF3, but not AA861, can prevent demyelination and axonal injury in mice after EAE induction.

AACOCF3 attenuated nitrotyrosine formation in the EAE mouse spinal cord white matter

Peroxynitrite, the highly toxic reaction product of nitric oxide and superoxide (Beckman and Koppenol, 1996), has been shown to contribute to the pathology found in EAE/MS (Hooper et al., 1997; Liu et al., 2001; Spitsin et al., 2001). To support this notion, scavengers or the decomposition catalysts of peroxynitrite are found to be efficacious in the treatment of EAE (Bolton et al., 2008; Cross et al., 2000; Hooper et al., 1998, 2000). Here we tested whether the protective effects of AACOCF3 can be, at least in part, due to its inhibition of peroxynitrite formation. Using nitrotyrosine immunostaining, a marker for peroxynitrite formation, we found nitrotyrosine immunoreactivity was dramatically increased in the EAE mouse spinal cord white matter (Fig. 7), and almost completely blocked by the treatment with AACOCF3. This result strongly suggests that AACOCF3 may protect OLs and axons from injury via blocking peroxynitrite formation.

AACOCF3 blocked peroxynitrite formation in LPS-activated microglia and reactive microglia induced toxicity to mature OLs

We and others have previously shown that peroxynitrite is the major toxic species released from reactive microglia causing toxicity to neurons and mature OLs (Li et al., 2005; Xie et al., 2002; Zhang et al.,
2004, 2006). To test whether AACOCF3 can affect the production of peroxynitrite in reactive microglia, generation of nitric oxide and superoxide was examined in LPS activated microglia in the presence of AACOCF3. LPS at $1 \mu \mathrm{g} / \mathrm{ml}$ induced upregulation of $\mathrm{CPLA}_{2}$, iNOS and components of the NADPH oxidase complex, p47phox and p67phox, in a time-dependent manner. At 6-8 h following LPS treatment, the expression of $\mathrm{cPLA}_{2}$, iNOS, p47phox and p67phox was dramatically increased (Fig. 8A). Following $24 \mathrm{~h}$ LPS treatment, cPLA 2 and p67phox were found to be translocated to the plasma membrane (Fig. 8B), indicating that $\mathrm{CPLA}_{2}$ and NADPH oxidase are activated in LPSactivated microglia. AACOCF3 at $10 \mu \mathrm{M}$ attenuated upregulation of iNOS and p67phox in LPS activated microglia (Fig. 8C) and generation of nitric oxide and superoxide (Fig. 8D-E). In mixed cultures of microglia and mature OLs, LPS $(5 \mu \mathrm{g} / \mathrm{ml})$ caused toxicity to OLs, but not to microglia (Fig. 9). Similar to the effects of the peroxynitrite decomposition catalyst FeTMPyP, AACOCF3 also significantly attenuated cell death triggered by reactive microglia (Fig. 9). These results suggested that the protective effects of AACOCF3 might be attributable to its blockade of peroxynitrite formation.

\section{Discussion}

Inhibition of PLA $A_{2}$ has been shown to be effective for the treatment of EAE (Kalyvas et al., 2009; Kalyvas and David, 2004; Marusic et al., $2005,2008)$. However, the protective mechanisms of the pharmacological PLA $\mathrm{A}_{2}$ inhibitors are still unclear. In the present study, we found that administration of AACOCF3 after EAE induction reduced clinical scores and the typical weight loss of EAE mice. AACOCF3 markedly attenuated the loss of mature OLs and the injury to axons typically found in EAE. The protective effects of AACOCF3 are likely due to its improvement of the $\mathrm{BBB}$ integrity, its reduction of infiltrating leukocytes, and its inhibition of nitrotyrosine formation in the spinal cord white matter. In primary cultures of microglia, AACOCF3 attenuated iNOS induction, nitric oxide production and the generation of superoxide. Similar to the protective effect of FeTMPyP, a decomposition catalyst of peroxynitrite, AACOCF3 also significantly attenuated the toxicity of mature OLs triggered by reactive microglia.

Deregulation of lipid metabolism has been implicated in many inflammatory and neurodegenerative diseases, including MS, stroke, traumatic brain and spinal cord injury, and Alzheimer's disease (Adibhatla and Hatcher, 2008). Due to the high lipid contents in the central nervous system (CNS), hydrolysis of membrane phospholipids catalyzed by $\mathrm{PLA}_{2}$ might be important for initiation and progression of the aforementioned diseases. Although iPLA 2 and SPLA 2 are able to liberate arachidonic acids from membrane phospholipids (Balsinde and Balboa, 2005; Kudo and Murakami, 2002; Wijewickrama et al.,
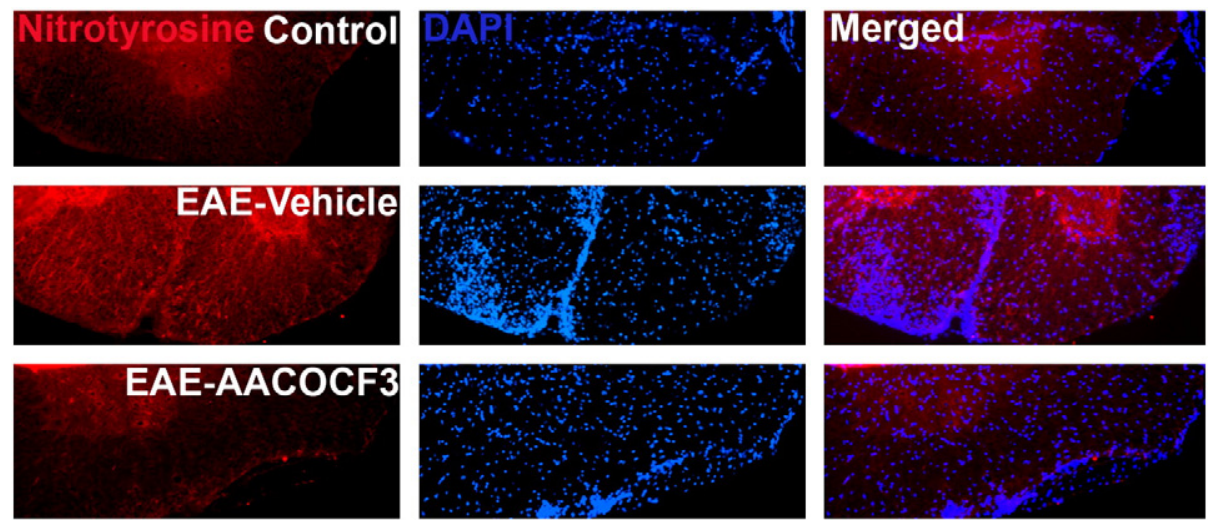

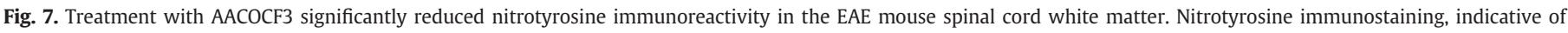

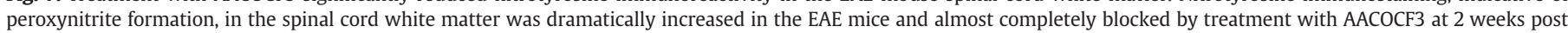
immunization. The images are representative of 6 different animals in each group. 
A
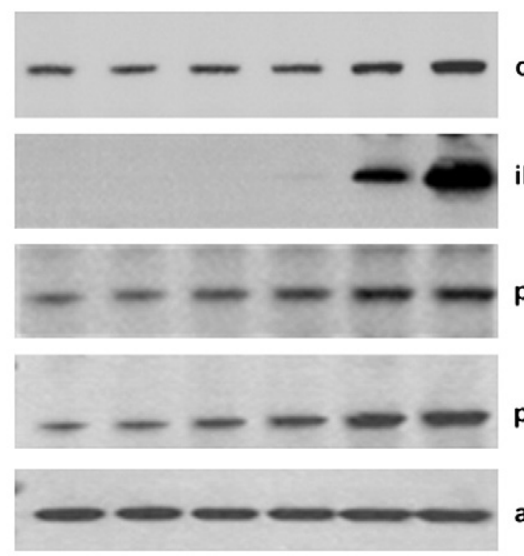

0

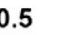

2

4

C
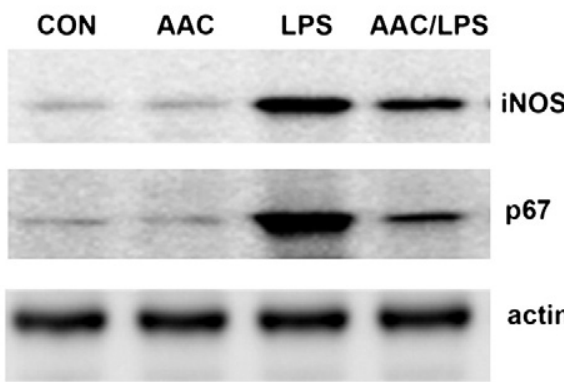

actin
B
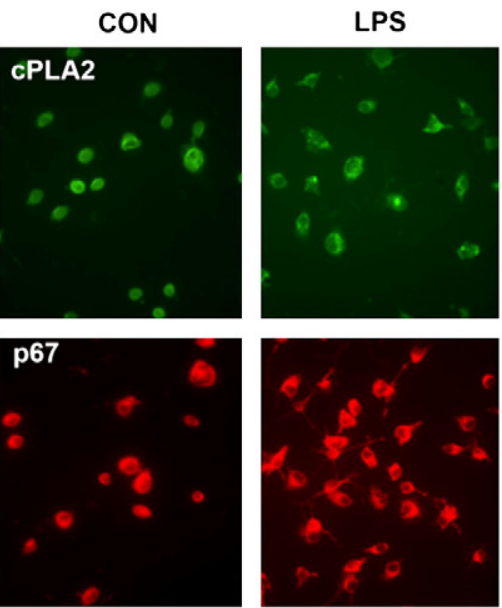

D

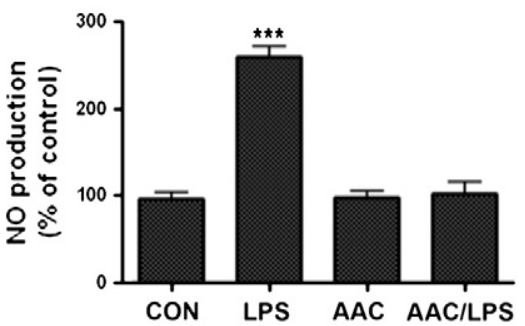

E

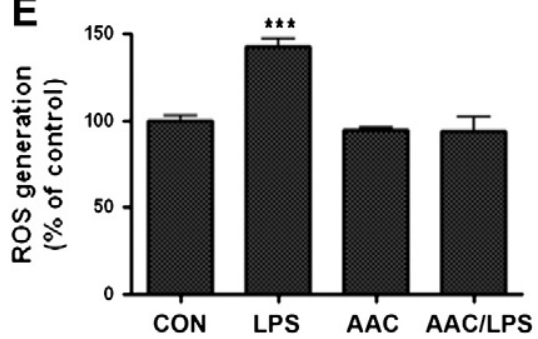

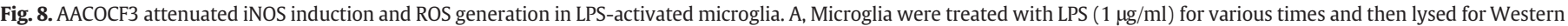

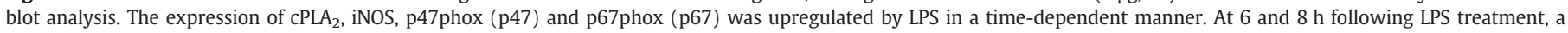

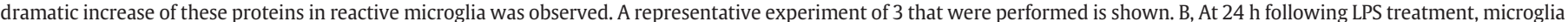

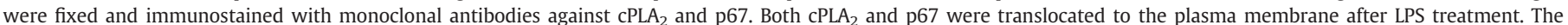

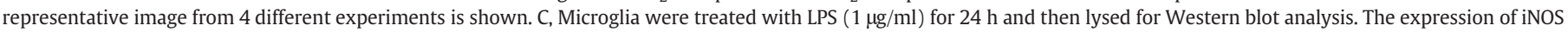

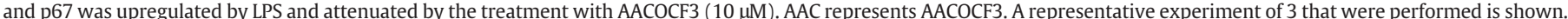

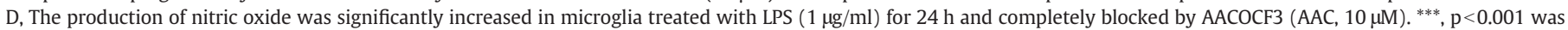

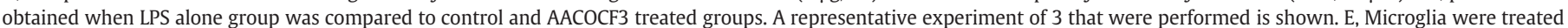

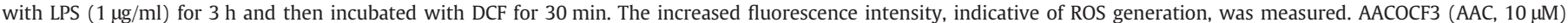

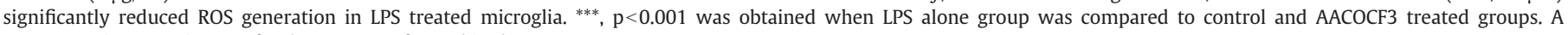
representative experiment of 3 that were performed is shown.

$2006 a, b), c P L A_{2} \alpha$ is thought to be the primary and the only isoform to selectively cleave arachidonyl phospholipids liberating arachidonic acids (Kita et al., 2006; Kudo and Murakami, 2002). The released arachidonic acids are in turn converted to prostaglandins and leukotrienes via activation of the COX and LOX pathways, respectively. These proinflammatory eicosanoids have long been suggested to contribute to the pathology of EAE (Bolton et al., 1984; Prosiegel et al., 1989; Reder et al., 1995), but the results obtained using pharmacological and genetic approaches are rather controversial. It has been shown that several COX and LOX inhibitors can reduce the severity of EAE (Marusic et al., 2008; Muthian et al., 2006; Ni et al., 2007; Ospelt et al., 2008). However, COX2 $2^{-1-}$ mice are found to develop EAE similar to the wild type controls (Miyamoto et al., 2006), and the disease severity is exacerbated in both $12-\mathrm{LOX}^{-1-}$ and 5 - $\mathrm{LOX}^{-1-}$ animals (Emerson and LeVine, 2004). The fact that rofecoxib, a COX inhibitor, was withdrawn due to its severe side effects in MS patients (Juni et al., 2004) suggests that inhibition of the COX pathway would not be clinically useful for the treatment of MS (James et al., 2007). It is also possible that inhibition of COX renders arachidonic acids to shift towards the LOX metabolic pathway, which can mediate OL toxicity and axonal injury. In addition to producing arachidonic acids, activation of $\mathrm{CPLA}_{2}$ also generates lysophosphatidylcholine and platelet-activating factors causing inflammation and demyelination in MS (Kihara et al., 2005; Kihara et al., 2008). All these findings indicate that CPLA $A_{2}$ may be a better target for EAE treatment. Indeed, we found that AACOCF3, the CPLA 2 inhibitor, but not AA861, the LOX inhibitor, reduced the severity of clinical symptoms in EAE. Addition of AA861 did not alter the protective effects of AACOCF3. In contrast to the recent report that ziluton, a 5-LOX inhibitor, delays EAE onset, but not the maximal severity of EAE (Marusic et al., 2008), we found 
A
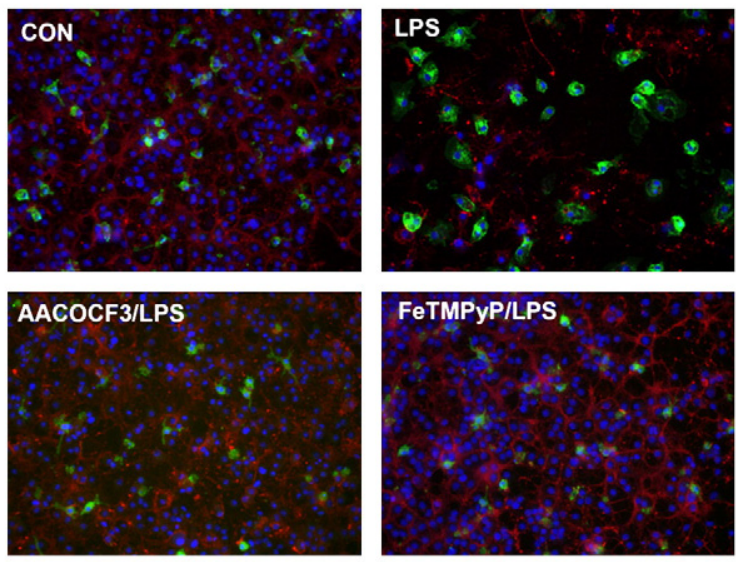

B

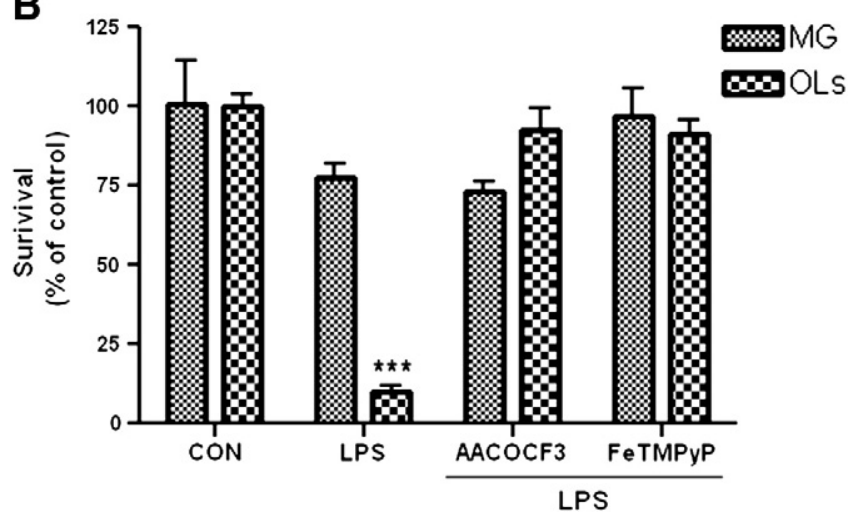

Fig. 9. AACOCF3 attenuated OL toxicity triggered by reactive microglia. A, Mixed cultures of microglia and mature OLs were treated with LPS $(5 \mu \mathrm{g} / \mathrm{ml})$ for $48 \mathrm{~h}$, then fixed and immunostained with F4/80 (green) and MBP (red). Nuclei were stained with DAPI (blue). The representative image from 3 different experiments is shown. B Survival of microglia and mature OLs was determined by counting F4/80 and $\mathrm{MBP}^{+}$ cells with normal nuclei at $48 \mathrm{~h}$ following LPS treatment. Five random fields were counted in each coverslip under $20 \times$ magnification. ${ }^{* * *}, \mathrm{p}<0.001$ was obtained when the numbers of $\mathrm{MBP}^{+}$OLs in LPS alone group were compared to those of the control and drug treated groups. The results were pooled from 3 different experiments.

AA861 neither affected the disease onset nor the maximal severity, although there was a trend for reduced clinical scores at the early onset of the disease. The similar result was also found when baicalein, a 12/15-LOX inhibitor (van Leyen et al., 2006), was administered (data not shown). It is unclear what causes the differences, but the factors may include the different dosages, routes of administration, and the characteristics of these inhibitors. It is shown that the protection afforded by the LOX inhibitor in the previous report is found to be much less than the COX and $\mathrm{CPLA}_{2}$ inhibitors (Marusic et al., 2008).

Although EAE is known to be a $\mathrm{T}$ cell-mediated disease, macrophage and microglia are likely the effecter cells causing toxicity to OLs, demyelination and axonal injury. Several studies have previously reported that $\mathrm{PLA}_{2}$ inhibition can diminish the Th1 and Th17 response and the production of inflammatory cytokines and chemokines (Bettelli et al., 2007; Kalyvas and David, 2004; Marusic et al., 2008), however, the role of $\mathrm{PLA}_{2}$ on the function of the infiltrating macrophage and the activated microglia in EAE are largely unknown. Similar to the findings by others (Kalyvas et al., 2009; Kalyvas and David, 2004), we found that the expression of CPLA $\mathrm{C}_{2}$ is elevated in macrophage and microglia in the EAE mouse spinal cord white matter. Although $\mathrm{CPLA}_{2}$ is also expressed in neurons and OLs, the differences of its expression in these cell types were not found between the EAE and control animals. Consistent with the reports that peroxynitrite may be an important contributor to the development of EAE (Hooper et al., 1997; Liu et al., 2001; Spitsin et al., 2001), we found that nitrotyrosine immunoreactivity was dramatically increased in the spinal cord white matter of EAE mice and almost completely blocked by treatment with AACOCF3. Similarly, we found AACOCF3 attenuated iNOS induction, the upregulation and translocation of p47phox and p67phox in LPS activated microglia. Since NADPH oxidase is believed to be the major enzyme for superoxide production in reactive microglia (Block et al., 2007; Li et al., 2005; Qin et al., 2004; Sun et al., 2007; Xie et al., 2002), these results suggest that AACOCF3 can attenuate the generation of nitric oxide and superoxide, and even more importantly, their highly toxic reaction product peroxynitrite. Furthermore, we found that similar to the protective effects of the decomposition catalyst of peroxynitrite, AACOCF3 significantly attenuated the toxicity of mature OLs triggered by reactive microglia, suggesting the protective action of AACOCF3 in EAE mice might be due to its inhibition of peroxynitrite formation by reactive microglia in the spinal cord white matter. Alternatively, AACOCF3 may produce immunomodulatory effects on peripheral leukocytes, thus inhibiting extravasation of these immune cells into the CNS parenchyma. This is at least partially supported by our findings that treatment with AACOCF3 preserves the BBB integrity in EAE mice.

It is known that AACOCF3 not only inhibits $\mathrm{CPLA}_{2}$, but also inhibits iPLA $_{2}$ and COX-2 (Ghomashchi et al., 1999; Kalyvas and David, 2004). Recently, it has been shown that the inhibitors of $\mathrm{CPLA}_{2}, \mathrm{iPLA}_{2}$ and $\mathrm{SPLA}_{2}$ can all reduce the severity of EAE, and activation of different $\mathrm{PLA}_{2}$ isoforms may be involved in the different stages of the disease (Kalyvas et al., 2009). However, except for $\mathrm{CPLA}_{2} \alpha$, the role of $\mathrm{PLLA}_{2}$ and SPLA ${ }_{2}$ in the development of EAE has not been validated by using genetic approaches. Nevertheless, these studies may help to develop isoform specific PLA 2 inhibitors for the treatment of EAE, and more importantly may offer valuable therapeutics for MS.

\section{Acknowledgments}

This work was supported by grants from the Blast Lethality Injury and Research Program (R600-070-00000-00-106109), the National Multiple Sclerosis Society (RG3741) and the Uniformed Services University (R070 UX and R0701Z). The authors wish to express their thanks and appreciation to Omar Logue and Dr. Fengshan Yu for their technical help.

\section{References}

Adibhatla, R.M., Hatcher, J.F., 2008. Altered lipid metabolism in brain injury and disorders. Subcell. Biochem. 49, 241-268.

Alvarez, J.I., Cayrol, R., Prat, A., 2011. Disruption of central nervous system barriers in multiple sclerosis. Biochim. Biophys. Acta 1812, 252-264.

Balsinde, J., Balboa, M.A., 2005. Cellular regulation and proposed biological functions of group VIA calcium-independent phospholipase A2 in activated cells. Cell. Signal. 17, 1052-1062.

Baud, O., Li, J., Zhang, Y., Neve, R.L., Volpe, J.J., Rosenberg, P.A., 2004. Nitric oxideinduced cell death in developing oligodendrocytes is associated with mitochondrial dysfunction and apoptosis-inducing factor translocation. Eur. J. Neurosci. 20, 1713-1726.

Beckman, J.S., Koppenol, W.H., 1996. Nitric oxide, superoxide, and peroxynitrite: the good, the bad, and ugly. Am. J. Physiol. 271, C1424-1437.

Bettelli, E., Oukka, M., Kuchroo, V.K., 2007. T(H)-17 cells in the circle of immunity and autoimmunity. Nat. Immunol. 8, 345-350.

Bjartmar, C., Wujek, J.R., Trapp, B.D., 2003. Axonal loss in the pathology of MS: consequences for understanding the progressive phase of the disease. J. Neurol. Sci. 206, 165-171.

Block, M.L., Zecca, L., Hong, J.S., 2007. Microglia-mediated neurotoxicity: uncovering the molecular mechanisms. Nat. Rev. Neurosci. 8, 57-69.

Bolton, C., Gordon, D., Turk, J.L., 1984. Prostaglandin and thromboxane levels in central nervous system tissues from rats during the induction and development of experimental allergic encephalomyelitis (EAE). Immunopharmacology 7, 101-107.

Bolton, C., Scott, G.S., Smith, T., Flower, R.J., 2008. The acute and chronic phases of chronic relapsing experimental autoimmune encephalomyelitis (CR EAE) are ameliorated by the peroxynitrite decomposition catalyst, 5,10,15,20-tetrakis(4- 
sulfonatophenyl)porphyrinatoiron (III) chloride, (FeTPPS). Eur. J. Pharmacol. 601, 88-93.

Cayrol, R., Wosik, K., Berard, J.L., Dodelet-Devillers, A., Ifergan, I., Kebir, H., Haqqani, A.S. Kreymborg, K., Krug, S., Moumdjian, R., Bouthillier, A., Becher, B., Arbour, N., David, S. Stanimirovic, D., Prat, A, 2008. Activated leukocyte cell adhesion molecule promotes leukocyte trafficking into the central nervous system. Nat. Immunol. 9, 137-145.

Chaudhary, P., Marracci, G.H., Bourdette, D.N., 2006. Lipoic acid inhibits expression of ICAM- 1 and VCAM- 1 by CNS endothelial cells and T cell migration into the spinal cord in experimental autoimmune encephalomyelitis. J. Neuroimmunol. 175, 87-96.

Cross, A.H., San, M., Stern, M.K., Keeling, R.M., Salvemini, D., Misko, T.P., 2000. A catalyst of peroxynitrite decomposition inhibits murine experimental autoimmune encephalomyelitis. J. Neuroimmunol. 107, 21-28.

Emerson, M.R., LeVine, S.M., 2004. Experimental allergic encephalomyelitis is exacerbated in mice deficient for 12/15-lipoxygenase or 5-lipoxygenase. Brain Res. 1021, 140-145.

Farooqui, A.A., Horrocks, L.A., 2006. Phospholipase A2-generated lipid mediators in the brain: the good, the bad, and the ugly. Neuroscientist 12, 245-260.

Farooqui, A.A., Ong, W.Y., Horrocks, L.A., 2006. Inhibitors of brain phospholipase A2 activity: their neuropharmacological effects and therapeutic importance for the treatment of neurologic disorders. Pharmacol. Rev. 58, 591-620.

Funk, C.D., 2001. Prostaglandins and leukotrienes: advances in eicosanoid biology. Science 294, 1871-1875.

Furlan, R., Cuomo, C., Martino, G., 2009. Animal models of multiple sclerosis. Methods Mol. Biol. 549, 157-173.

Ghomashchi, F., Loo, R., Balsinde, J., Bartoli, F., Apitz-Castro, R., Clark, J.D., Dennis, E.A. Gelb, M.H., 1999. Trifluoromethyl ketones and methyl fluorophosphonates as inhibitors of group IV and VI phospholipases A(2): structure-function studies with vesicle, micelle, and membrane assays. Biochim. Biophys. Acta 1420, 45-56.

Hooper, D.C., Bagasra, O., Marini, J.C., Zborek, A., Ohnishi, S.T., Kean, R., Champion, J.M. Sarker, A.B., Bobroski, L., Farber, J.L., Akaike, T., Maeda, H., Koprowski, H., 1997. Prevention of experimental allergic encephalomyelitis by targeting nitric oxide and peroxynitrite: implications for the treatment of multiple sclerosis. Proc. Natl. Acad. Sci. U. S. A. 94, 2528-2533.

Hooper, D.C., Spitsin, S., Kean, R.B., Champion, J.M., Dickson, G.M., Chaudhry, I., Koprowski, H., 1998. Uric acid, a natural scavenger of peroxynitrite, in experimental allergic encephalomyelitis and multiple sclerosis. Proc. Natl. Acad. Sci. U. S. A. 95, 675-680.

Hooper, D.C., Scott, G.S., Zborek, A., Mikheeva, T., Kean, R.B., Koprowski, H., Spitsin, S.V., 2000. Uric acid, a peroxynitrite scavenger, inhibits CNS inflammation, blood-CNS barrier permeability changes, and tissue damage in a mouse model of multiple sclerosis. FASEB J. 14, 691-698.

James, M.J., Cook-Johnson, R.J., Cleland, L.G., 2007. Selective COX-2 inhibitors, eicosanoid synthesis and clinical outcomes: a case study of system failure. Lipids 42, 779-785.

Juni, P., Nartey, L., Reichenbach, S., Sterchi, R., Dieppe, P.A., Egger, M., 2004. Risk of cardiovascular events and rofecoxib: cumulative meta-analysis. Lancet 364 2021-2029.

Kalyvas, A., David, S., 2004. Cytosolic phospholipase A2 plays a key role in the pathogenesis of multiple sclerosis-like disease. Neuron 41, 323-335.

Kalyvas, A., Baskakis, C., Magrioti, V., Constantinou-Kokotou, V., Stephens, D., LopezVales, R., Lu, J.Q., Yong, V.W., Dennis, E.A., Kokotos, G., David, S., 2009. Differing roles for members of the phospholipase A2 superfamily in experimental autoimmune encephalomyelitis. Brain 132, 1221-1235.

Kihara, Y., Ishii, S., Kita, Y., Toda, A., Shimada, A., Shimizu, T., 2005. Dual phase regulation of experimental allergic encephalomyelitis by platelet-activating factor. J. Exp. Med. 202, 853-863.

Kihara, Y., Yanagida, K., Masago, K., Kita, Y., Hishikawa, D., Shindou, H., Ishii, S., Shimizu, T., 2008. Platelet-activating factor production in the spinal cord of experimental allergic encephalomyelitis mice via the group IVA cytosolic phospholipase A2-lysoPAFAT axis. J. Immunol. 181, 5008-5014.

Kita, Y., Ohto, T., Uozumi, N., Shimizu, T., 2006. Biochemical properties and pathophysiological roles of cytosolic phospholipase A2s. Biochim. Biophys. Acta $1761,1317-1322$

Kudo, I., Murakami, M., 2002. Phospholipase A2 enzymes. Prostaglandins Other Lipid Mediat. 68-69, 3-58.

Lassmann, H., 2010. Axonal and neuronal pathology in multiple sclerosis: what have we learnt from animal models. Exp. Neurol. 225, 2-8.

Li, J., Baud, O., Vartanian, T., Volpe, J.J., Rosenberg, P.A., 2005. Peroxynitrite generated by inducible nitric oxide synthase and NADPH oxidase mediates microglial toxicity to oligodendrocytes. Proc. Natl. Acad. Sci. U. S. A. 102, 9936-9941.

Liu, J.S., Zhao, M.L., Brosnan, C.F., Lee, S.C., 2001. Expression of inducible nitric oxide synthase and nitrotyrosine in multiple sclerosis lesions. Am. J. Pathol. 158 2057-2066.

Liu, N.K., Zhang, Y.P., Titsworth, W.L., Jiang, X., Han, S., Lu, P.H., Shields, C.B., Xu, X.M., 2006. A novel role of phospholipase A2 in mediating spinal cord secondary injury. Ann. Neurol. 59, 606-619.
Marusic, S., Leach, M.W., Pelker, J.W., Azoitei, M.L., Uozumi, N., Cui, J., Shen, M.W., DeClercq, C.M., Miyashiro, J.S., Carito, B.A., Thakker, P., Simmons, D.L., Leonard, J.P., Shimizu, T., Clark, J.D., 2005. Cytosolic phospholipase A2 alpha-deficient mice are resistant to experimental autoimmune encephalomyelitis. J. Exp. Med. 202, 841-851.

Marusic, S., Thakker, P., Pelker, J.W., Stedman, N.L., Lee, K.L., McKew, J.C., Han, L., Xu, X Wolf, S.F., Borey, A.J., Cui, J., Shen, M.W., Donahue, F., Hassan-Zahraee, M., Leach, M.W., Shimizu, T., Clark, J.D., 2008. Blockade of cytosolic phospholipase A2 alpha prevents experimental autoimmune encephalomyelitis and diminishes development of Th1 and Th17 responses. J. Neuroimmunol. 204, 29-37.

Miyamoto, K., Miyake, S., Mizuno, M., Oka, N., Kusunoki, S., Yamamura, T., 2006. Selective COX-2 inhibitor celecoxib prevents experimental autoimmune encephalomyelitis through COX-2-independent pathway. Brain 129, 1984-1992.

Muthian, G., Raikwar, H.P., Johnson, C., Rajasingh, J., Kalgutkar, A., Marnett, L.J., Bright, J.J., 2006. COX-2 inhibitors modulate IL-12 signaling through JAK-STAT pathway leading to Th1 response in experimental allergic encephalomyelitis. J. Clin. Immunol. 26, 73-85.

Ni, J., Shu, Y.Y., Zhu, Y.N., Fu, Y.F., Tang, W., Zhong, X.G., Wang, H., Yang, Y.F., Ren, J., Wang, M.W., Zuo, J.P., 2007. COX-2 inhibitors ameliorate experimental autoimmune encephalomyelitis through modulating IFN-gamma and IL-10 production by inhibiting T-bet expression. J. Neuroimmunol. 186, 94-103.

Ospelt, C., Kurowska-Stolarska, M., Neidhart, M., Michel, B.A., Gay, R.E., Laufer, S., Gay, S., 2008. The dual inhibitor of lipoxygenase and cyclooxygenase ML3000 decreases the expression of CXCR3 ligands. Ann. Rheum. Dis. 67, 524-529.

Prosiegel, M., Neu, I., Mallinger, J., Wildfeuer, A., Mehlber, L., Vogl, S., Hoffmann, G., Ruhenstroth-Bauer, G., 1989. Suppression of experimental autoimmune encephalomyelitis by dual cyclo-oxygenase and 5-lipoxygenase inhibition. Acta Neurol. Scand. 79, 223-226.

Qin, L., Liu, Y., Wang, T., Wei, S.J., Block, M.L., Wilson, B., Liu, B., Hong, J.S., 2004. NADPH oxidase mediates lipopolysaccharide-induced neurotoxicity and proinflammatory gene expression in activated microglia. J. Biol. Chem. 279, 1415-1421.

Reder, A.T., Thapar, M., Sapugay, A.M., Jensen, M.A., 1995. Eicosenoids modify experimental allergic encephalomyelitis. Am. J. Ther. 2, 711-720.

Sanchez-Mejia, R.O., Newman, J.W., Toh, S., Yu, G.Q., Zhou, Y., Halabisky, B., Cisse, M. Scearce-Levie, K., Cheng, I.H., Gan, L., Palop, J.J., Bonventre, J.V., Mucke, L., 2008. Phospholipase A2 reduction ameliorates cognitive deficits in a mouse model of Alzheimer's disease. Nat. Neurosci. 11, 1311-1318.

Shelat, P.B., Chalimoniuk, M., Wang, J.H., Strosznajder, J.B., Lee, J.C., Sun, A.Y., Simonyi, A., Sun, G.Y., 2008. Amyloid beta peptide and NMDA induce ROS from NADPH oxidase and AA release from cytosolic phospholipase A2 in cortical neurons. J. Neurochem. 106, 45-55.

Spitsin, S., Hooper, D.C., Leist, T., Streletz, L.J., Mikheeva, T., Koprowskil, H., 2001. Inactivation of peroxynitrite in multiple sclerosis patients after oral administration of inosine may suggest possible approaches to therapy of the disease. Mult. Scler. 7, 313-319.

Steinman, L., Zamvil, S.S., 2006. How to successfully apply animal studies in experimental allergic encephalomyelitis to research on multiple sclerosis. Ann. Neurol. 60, 12-21.

Sun, G.Y., Horrocks, L.A., Farooqui, A.A., 2007. The roles of NADPH oxidase and phospholipases A2 in oxidative and inflammatory responses in neurodegenerative diseases. J. Neurochem. 103, 1-16.

Trapp, B.D., Peterson, J., Ransohoff, R.M., Rudick, R., Mork, S., Bo, L., 1998. Axonal transection in the lesions of multiple sclerosis. N. Engl. J. Med. 338, 278-285.

van Leyen, K., Kim, H.Y., Lee, S.R., Jin, G., Arai, K., Lo, E.H., 2006. Baicalein and 12/15lipoxygenase in the ischemic brain. Stroke 37, 3014-3018.

Wang, H., Li, J., Follett, P.L., Zhang, Y., Cotanche, D.A., Jensen, F.E., Volpe, J.J., Rosenberg, P.A., 2004. 12-Lipoxygenase plays a key role in cell death caused by glutathione depletion and arachidonic acid in rat oligodendrocytes. Eur. J. Neurosci. 20, 2049-2058.

Wijewickrama, G.T., Albanese, A., Kim, Y.J., Oh, Y.S., Murray, P.S., Takayanagi, R., Tobe, T., Masuda, S., Murakami, M., Kudo, I., Ucker, D.S., Murray, D., Cho, W., 2006a. Unique membrane interaction mode of group IIF phospholipase A2. J. Biol. Chem. 281, 32741-32754.

Wijewickrama, G.T., Kim, J.H., Kim, Y.J., Abraham, A., Oh, Y., Ananthanarayanan, B., Kwatia, M., Ackerman, S.J., Cho, W., 2006b. Systematic evaluation of transcellular activities of secretory phospholipases A2. High activity of group V phospholipases A2 to induce eicosanoid biosynthesis in neighboring inflammatory cells. J. Biol. Chem. 281, 10935-10944

Xie, Z., Wei, M., Morgan, T.E, Fabrizio, P., Han, D., Finch, C.E., Longo, V.D., 2002. Peroxynitrite mediates neurotoxicity of amyloid beta-peptide1-42- and lipopolysaccharide-activated microglia. J. Neurosci. 22, 3484-3492.

Zhang, Y., Wang, H., Li, J., Jimenez, D.A., Levitan, E.S., Aizenman, E., Rosenberg, P.A., 2004. Peroxynitrite-induced neuronal apoptosis is mediated by intracellular zinc release and 12-lipoxygenase activation. J. Neurosci. 24, 10616-10627.

Zhang, Y., Wang, H., Li, J., Dong, L., Xu, P., Chen, W., Neve, R.L., Volpe, J.J., Rosenberg, P.A., 2006. Intracellular zinc release and ERK phosphorylation are required upstream of 12-lipoxygenase activation in peroxynitrite toxicity to mature rat oligodendrocytes. J. Biol. Chem. 281, 9460-9470. 